\title{
Disorder-specific and shared neurophysiological impairments of attention and inhibition in women with attention-deficit/hyperactivity disorder and women with bipolar disorder
}

\author{
G. Michelini ${ }^{1}$, G. L. Kitsune ${ }^{1,2}$, G. M. Hosang ${ }^{3}$, P. Asherson ${ }^{1}$, G. McLoughlin ${ }^{1}$ and J. Kuntsi ${ }^{1 *}$ \\ ${ }^{1}$ King's College London, MRC Social, Genetic and Developmental Psychiatry Centre, Institute of Psychiatry, Psychology and Neuroscience, \\ London, UK \\ ${ }^{2}$ Department of Psychological Medicine, King's College London, Institute of Psychiatry, Psychology and Neuroscience, London, UK \\ ${ }^{3}$ Department of Psychology, Goldsmiths, University of London, London, UK
}

\begin{abstract}
Background. In adults, attention-deficit/hyperactivity disorder (ADHD) and bipolar disorder (BD) have certain overlapping symptoms, which can lead to uncertainty regarding the boundaries of the two disorders. Despite evidence of cognitive impairments in both disorders separately, such as in attentional and inhibitory processes, data on direct comparisons across ADHD and BD on cognitive-neurophysiological measures are as yet limited.
\end{abstract}

Method. We directly compared cognitive performance and event-related potential measures from a cued continuous performance test in 20 women with ADHD, 20 women with BD (currently euthymic) and 20 control women.

Results. The NoGo-N2 was attenuated in women with BD, reflecting reduced conflict monitoring, compared with women with ADHD and controls (both $p<0.05$ ). Both ADHD and BD groups showed a reduced NoGo-P3, reflecting inhibitory control, compared with controls (both $p<0.05$ ). In addition, the contingent negative variation was significantly reduced in the ADHD group ( $p=0.05)$, with a trend in the BD group ( $p=0.07)$, compared with controls.

Conclusions. These findings indicate potential disorder-specific (conflict monitoring) and overlapping (inhibitory control, and potentially response preparation) neurophysiological impairments in women with ADHD and women with BD. The identified neurophysiological parameters further our understanding of neurophysiological impairments in women with $\mathrm{ADHD}$ and $\mathrm{BD}$, and are candidate biomarkers that may aid in the identification of the diagnostic boundaries of the two disorders.

Received 18 February 2015; Revised 24 August 2015; Accepted 27 August 2015; First published online 10 November 2015

Key words: Attention, attention-deficit/hyperactivity disorder, bipolar disorder, conflict monitoring, event-related potentials, inhibitory control.

\section{Introduction}

Attention-deficit/hyperactivity disorder (ADHD) and bipolar disorder (BD) are common psychiatric conditions in adults, affecting around $2-4 \%$ and $1-2 \%$ of the adult population, respectively (Merikangas et al. 2011; Willcutt, 2012). Although ADHD and BD represent distinct conditions, their diagnostic formulations present certain areas of symptomatic overlap. In adults, ADHD may be manifest with some symptoms common to mania/hypomania, such as distractibility,

* Address for correspondence: J. Kuntsi, Ph.D., MRC Social, Genetic and Developmental Psychiatry Centre, Institute of Psychiatry, Psychology and Neuroscience, King's College London, De Crespigny Park, London SE5 8AF, UK.

(Email: jonna.kuntsi@kcl.ac.uk) psychomotor restlessness and talkativeness (Skirrow et al. 2012; Asherson et al. 2014). Additionally, both disorders are associated with features of mood dysregulation, such as irritability and emotional lability (Skirrow et al. 2012, 2014; GL Kitsune et al. unpublished observations). Of note, ADHD symptoms are chronic and traitlike, while BD symptoms of mania and depression tend to occur for a distinct period of time (Asherson et al. 2014). Yet, individuals with BD may still show residual symptoms of distractibility and mood dysregulation (overlapping with ADHD), and residual cognitive and functional impairments between episodes (Torres et al. 2007; Henry et al. 2013). Importantly, symptomatic similarities can result in uncertainty regarding the boundaries of the two disorders, and difficulties in distinguishing between the two disorders in some patients, which in turn may result in inappropriate treatment decisions (Asherson et al. 2014). 
Adults with ADHD or BD may display similar cognitive impairments. For example, both ADHD and euthymic BD are associated with poor accuracy in attentional and inhibitory processing tasks (Robinson et al. 2006; McLoughlin et al. 2010; Torralva et al. 2011) and increased reaction time variability (RTV), which may reflect short-term fluctuations in attentional performance (Brotman et al. 2009; Kuntsi et al. 2010; Kuntsi \& Klein, 2012). Comparative studies across ADHD and BD, using identical measures, may aid in the identification of attentional and inhibitory deficits underlying overlapping symptoms and functional impairment, yet empirical data are currently limited.

The investigation of neurophysiological processes with event-related potentials (ERPs) provides a direct measure of covert brain activity underlying behavioural performance with millisecond temporal resolution, and may enable a sensitive comparison of cognitive profiles in ADHD and BD (Banaschewski \& Brandeis, 2007; McLoughlin et al. 2014a). Several previous studies on attentional and inhibitory processing in ADHD have explored ERPs during the cued continuous performance test (CPT-OX), which involves presentation of cue, target (Go) and non-target (NoGo) stimuli and requires a response only when a target follows a cue (van Leeuwen et al. 1998; Banaschewski et al. 2004). A reduced fronto-central P3 has consistently been reported in response to NoGo stimuli (NoGo-P3) in children, adolescents and adults with ADHD compared with controls, reflecting abnormal inhibitory control (Valko et al. 2009; Doehnert et al. 2010; McLoughlin et al. 2010, 2011; Albrecht et al. 2013; Tye et al. 2014). Attenuations in a parietal P3 after presentation of cue stimuli (Cue-P3) and in the subsequent contingent negative variation (CNV), a late negative potential before the occurrence of the next stimulus, have also been found in individuals with ADHD, reflecting impaired attentional orienting and response preparation, respectively (Doehnert et al. 2010; McLoughlin et al. 2010, 2011; Albrecht et al. 2013), although case-control differences in these components have not been reported in all studies (Dhar et al. 2010; Skirrow, 2012). Differences between adults with ADHD and control adults are generally not found in other ERP components elicited by this task; such as the P3 in response to target (Go-P3), reflecting response execution, and the $\mathrm{N} 2$ to non-target stimuli (NoGo-N2), indexing conflict monitoring, which refers to the ability to monitor ongoing behaviour, detect conflict and adjust response selection (Yeung \& Cohen, 2006; McLoughlin et al. 2010). N2 deflections are particularly elicited by high-conflict trials, such as non-target or incongruent stimuli, and are attenuated in ADHD individuals in paradigms inducing higher conflict-monitoring demands than the
CPT-OX, such as flanker tasks, suggesting possible modulations of this component by task and stimuli (Barry et al. 2009; McLoughlin et al. 2009, 2014b).

In ERP studies, BD has been associated with attenuations in early sensory and attentional ERP components (e.g. mismatch negativity, P50 and P2) in auditory tasks (Hall et al. 2007; Jahshan et al. 2012; Cabranes et al. 2013; Swann et al. 2013). Reduced P3 enhancements to target stimuli have been reported in adults with BD in studies using a visual paradigm with standard, deviant and target conditions (Maekawa et al. 2013) and using an oddball paradigm (Hall et al. 2007), but not in all studies (Schulze et al. 2008; Bestelmeyer, 2012). Some evidence also indicates impairments in conflict monitoring in adults with $\mathrm{BD}$, indexed by reduced N2 in response to target stimuli with an auditory oddball task (Ethridge et al. 2012) and reduced error-related negativity (ERN) in error responses (Morsel et al. 2014). Despite initial evidence that may suggest impairments in ERPs of attentional and inhibitory processing in $\mathrm{BD}$, however, ERP data on these processes are limited, and no studies, to our knowledge, have used the CPT-OX.

Direct comparisons on cognitive performance and ERP measures in ADHD and BD are sparse. One study on adults with ADHD and adults with BD investigating ERP measures of reward processing found significant differences in the amplitude of a rewardsensitive P3, which was attenuated in ADHD but enhanced in BD participants compared with controls (Ibanez et al. 2012). However, no study to date has compared ERP components associated with attentional and inhibitory processing in both disorders using the CPT-OX. In addition, most studies of this kind, especially on ADHD, have used male samples because, among children, ADHD is more prevalent in males than in females, and very little is known about these processes in females. Yet, a similar prevalence of ADHD has been reported in both adult men and women (Faraone \& Biederman, 2005; Das et al. 2012). Similarly, comparable gender ratios have been found for BD in adults (Pini et al. 2005).

The aim of the current study was to directly compare cognitive performance and ERP measures associated with attentional and inhibitory processing in ADHD and BD in adults. This study was conducted on an allfemale sample, in order to match the groups on gender but also to explore the neglected area of ERP indices associated with these processes in females. Based on previous studies of male participants (McLoughlin et al. 2010; Albrecht et al. 2013; Doehnert et al. 2013), we predicted that women with ADHD would show reduced NoGo-P3, Cue-P3 and CNV, but normal NoGo-N2. Given the limited and mixed results in ERP studies of BD individuals and the lack of similar 
studies using the CPT-OX, we adopted an exploratory approach for the $\mathrm{BD}$ group and for the comparison with ADHD.

\section{Method}

\section{Sample}

The sample for this study consisted of 60 adult women aged between 20 and 52 years, divided into three groups: 20 with ADHD, 20 with $\mathrm{BD}$ and 20 controls. Participants with ADHD were recruited from the National Adult ADHD Clinic at the Maudsley Hospital, where any female cases meeting inclusion criteria were considered for potential inclusion in the study. Participants with BD were recruited from the Maudsley Psychosis Clinic and a sample that had previously participated in another research study (Hosang et al. 2012). Control participants were recruited from the Mindsearch volunteer database maintained by the Institute of Psychiatry, King's College London, which comprises several thousand potential participants. Participants were randomly selected from all those meeting recruitment criteria for this study.

Diagnosis in the clinical groups was confirmed by checking medical records for details of diagnosis and psychiatric history, following Diagnostic and Statistical Manual of Mental Disorders, 4th edition (DSM-IV) criteria (APA, 2000). All of the ADHD participants had a current combined-type diagnosis or a current inattentive-type diagnosis with sufficient symptoms of hyperactivity-impulsivity in childhood to meet a childhood combined-type diagnosis. Participants in the BD group had a diagnosis of BD type I, having experienced at least one manic episode in the past. Those who were experiencing a manic episode at the time of the assessment were excluded; all participants included in the BD group were currently euthymic. Exclusion criteria for all groups were drug or alcohol dependency in the last 6 months, autism, epilepsy, neurological disorders, brain injury, past electroconvulsive therapy, current involvement in another research trial likely to alter symptom severity, pregnancy or a limited proficiency in the English language. Individuals with ADHD and individuals with BD with a reported co-morbidity of both ADHD and BD were also excluded. Control participants, who reported a history of psychiatric disorders or who were taking psychiatric medication, were excluded from the study. Co-morbidity in the clinical groups and lack of psychiatric disorders in the control group were further assessed through clinical evaluations when participants underwent the cognitive-electroencephalographic (EEG) assessment for this study. Further details on the clinical assessment of this sample can be found elsewhere (GL Kitsune et al. unpublished observations). In brief,
ADHD was excluded in the BD group after conducting the Diagnostic Interview for ADHD in Adults (DIVA v. 2.0; Kooij \& Francken, 2007). BD was excluded in the ADHD group by checking for a history of past episodes of depression or hypomania/mania and evaluating current mood symptoms using the Altman Self-Rating Mania Scale (Altman et al. 1997) and the Beck Depression Inventory (Beck et al. 1996), and current and lifetime ever symptoms using the Young Mania Rating Scale (Young et al. 1978). The ADHD and BD groups did not differ significantly on any of the mood scales for current symptoms (GL Kitsune et al. unpublished observations).

All participants had normal or corrected-to-normal vision. Mean age did not differ by group $\left(F_{2,59}=1.63\right.$, $p=0.21$ ), with a mean age of 37.40 (s.D. $=7.70$ ) for the ADHD group, 40.30 (s.D. =7.70) for the BD group and 36.7 (S.D. $=4.30$ ) for the control group. Participants' intelligence quotients (IQs) were assessed with the Wechsler Abbreviated Scale of Intelligence, fourth edition (Wechsler, 1999) and did not differ between groups $\left(F_{2,58}=1.37, p=0.26\right)$, with mean IQs of 104 (S.D. = 17.90) for ADHD, 108 (s.D.=12.50) for BD and 112 (S.D. $=14.20$ ) for control participants. Participants with ADHD were asked to stop taking any stimulant medication prescribed for their ADHD $48 \mathrm{~h}$ prior to the assessment. For ethical reasons, participants were not asked to stop taking mood stabilizers $(70 \%$ of the BD group), anti-psychotic medication (40\% of the BD group) or anti-depressants (7\% of the ADHD group and $25 \%$ of the BD group) they had been prescribed. All participants were asked to refrain from caffeinated drinks and nicotine $2 \mathrm{~h}$ prior to the testing session. Ethical approval for the study was granted by the Camberwell St Giles Research Ethics Committee (approval number 11/LO/0438) and all participants provided informed consent.

\section{Procedure and cognitive performance measures}

Participants attended a single $4.5 \mathrm{~h}$ research session (including breaks) for cognitive-EEG assessment, IQ assessment and clinical interviews. The task was a CPT-OX, flanker version (Doehnert et al. 2008; McLoughlin et al. 2010, 2011). This is a cued-Go/NoGo task that probes attention, preparation and response inhibition or control. The task consists of 400 letter arrays formed of a centre letter with incompatible flankers on each side to increase difficulty for adults. Each letter array was presented for $150 \mathrm{~ms}$ with a stimulus onset asynchrony (SOA) of 1.65 $\mathrm{s}$ in a pseudo-randomized order at the centre of a computer monitor. The tasks involved the presentation of 80 cues (XOX) followed either by $40 \mathrm{Go}(\mathrm{OXO})$ and 40 NoGo (XDX) stimuli, alternated with random letter sequences as distractors. Participants were instructed to 
respond only to Cue-Go sequences by pressing a button as quickly as possible with the digit finger of their preferred hand, and to withhold the response in presence of a NoGo stimulus, of a Go not preceded by a cue (40 trials), or of any other irrelevant letters. The task was practised prior to task performance and lasted $11 \mathrm{~min}$. The task followed a $2 \times 3 \mathrm{~min}$ restingstate recording, and was run as first in a battery of three cognitive-EEG tasks.

Cognitive performance measures included target mean reaction time (MRT, i.e. mean latency of responding in ms after target onset), RTV (measured as standard deviation of target reaction time) and number of errors. MRT and RTV were calculated across correctly answered Go trials. Errors included omission errors (non-response to Go trials), total commission errors (response to cue, NoGo or distractor stimuli) and OXO-not-XOX commission errors (response to a Go not following a cue).

\section{Electrophysiological recording and analysis}

The EEG was recorded from a 62-channel DC-coupled recording system (extended 10-20 montage), using a $500 \mathrm{~Hz}$ sampling rate, impedances under $10 \mathrm{k} \Omega$, and $\mathrm{FC} z$ as the recording reference. The electro-oculograms were recorded from electrodes above and below the left eye and at the outer canthi. The EEG data were analysed using Brain Vision Analyser 2.0 (Brain Products). Researchers were blind to group status during EEG pre-processing and analysis. Raw EEG recordings were down-sampled to $256 \mathrm{~Hz}$, re-referenced to the average of all electrodes, and digitally filtered using Butterworth band-pass filters $(0.1-30 \mathrm{~Hz}, 24$ $\mathrm{dB} /$ octave). All trials were also visually inspected for electrical artefacts (due to electrical noise in the EEG recording) or obvious movement, and sections of data containing artefacts were removed manually. Ocular artefacts, corresponding to blink-related and vertical and horizontal eye movements, were identified using the infomax Independent Component Analysis algorithm (ICA; Jung et al. 2000), which allows for removal of the components associated with ocular artefacts by back-projection of all but those components. Sections of data with remaining artefacts exceeding $\pm 100 \mu \mathrm{V}$ in any channel or with a voltage step greater than $50 \mu \mathrm{V}$ were automatically rejected. Baseline correction was performed using a 500-ms pre-stimulus reference period ${ }^{1}+$.

Stimulus-locked epochs (stimulus window from -200 to $1650 \mathrm{~ms}$ ) were averaged based on three different response conditions: cue, Go and NoGo. Averages

+ The notes appear after the main text. only included trials with correct responses (Go) or correctly rejected trials (NoGo and cue) and contained at least 20 artefact-free segments (see online Supplementary material for number of segments included in the ERP average by group). ERP measures were identified within the selected electrodes and latency windows for which effects were expected to be largest, based on previous studies (McLoughlin et al. 2010, 2011; Albrecht et al. 2013; Doehnert et al. 2013) and verified against the topographic maps and the grand averages (Figs 1-3). ERPs were measured as the mean amplitude in the designated latency window. This approach has been adopted in previous similar studies (Groom et al. 2010; Tye et al. 2014), and has the advantage of being unaffected by latency variability (Luck, 2005). In cue trials, the P3 was measured at $\mathrm{Pz}$ between 300 and $650 \mathrm{~ms}$, and the CNV at $\mathrm{Cz}$ between 1300 and 1650 ms. In NoGo trials, the N2 was measured at Fz between 175 and $325 \mathrm{~ms}$, and the P3 at Cz between 250 and 550 ms. In Go trials, the P3 was measured at $\mathrm{CPz}$ between 250 and $500 \mathrm{~ms}$. A clear N2 was not observed in Go trials, in line with other studies on tasks inducing a low-conflict-monitoring demand (Bokura et al. 2001; Gajewski \& Falkenstein, 2013) and was not included into the analysis.

\section{Statistical analyses}

All participants were included in the analysis of cognitive performance data. Two ADHD participants were excluded from the ERP analysis of the Go condition due to having less than 20 artefact-free segments available for analysis.

Group differences on the reaction time measures were explored using univariate analyses of variance (ANOVAs), followed by post-hoc $t$ tests. MRT and RTV had skewed distributions and were logtransformed with optimized minimal skew through the 'Inskew0' command in Stata (Stata Corp.). Performance accuracy was generally high as errors were rare, in line with previous studies on this task (McLoughlin et al. 2010, 2011; Albrecht et al. 2013; Doehnert et al. 2013). Since distribution of errors was thus not normal and no transformations were successful, effects of group on these variables were entered into non-parametric analysis, using Kruskal-Wallis tests, followed by post-hoc Mann-Whitney $U$ tests.

Group effects on ERP parameters were tested with separate ANOVAs, followed by post-hoc $t$ tests. All ERP measures had normal distribution. We report both $p$ values $(p<0.05$ for significance and $p<0.10$ for a trend) and effect sizes (Cohen's $d$ ) for comparisons of cognitive performance and ERP measures. Effect sizes were calculated using the difference in the means, divided by the pooled standard deviation, 


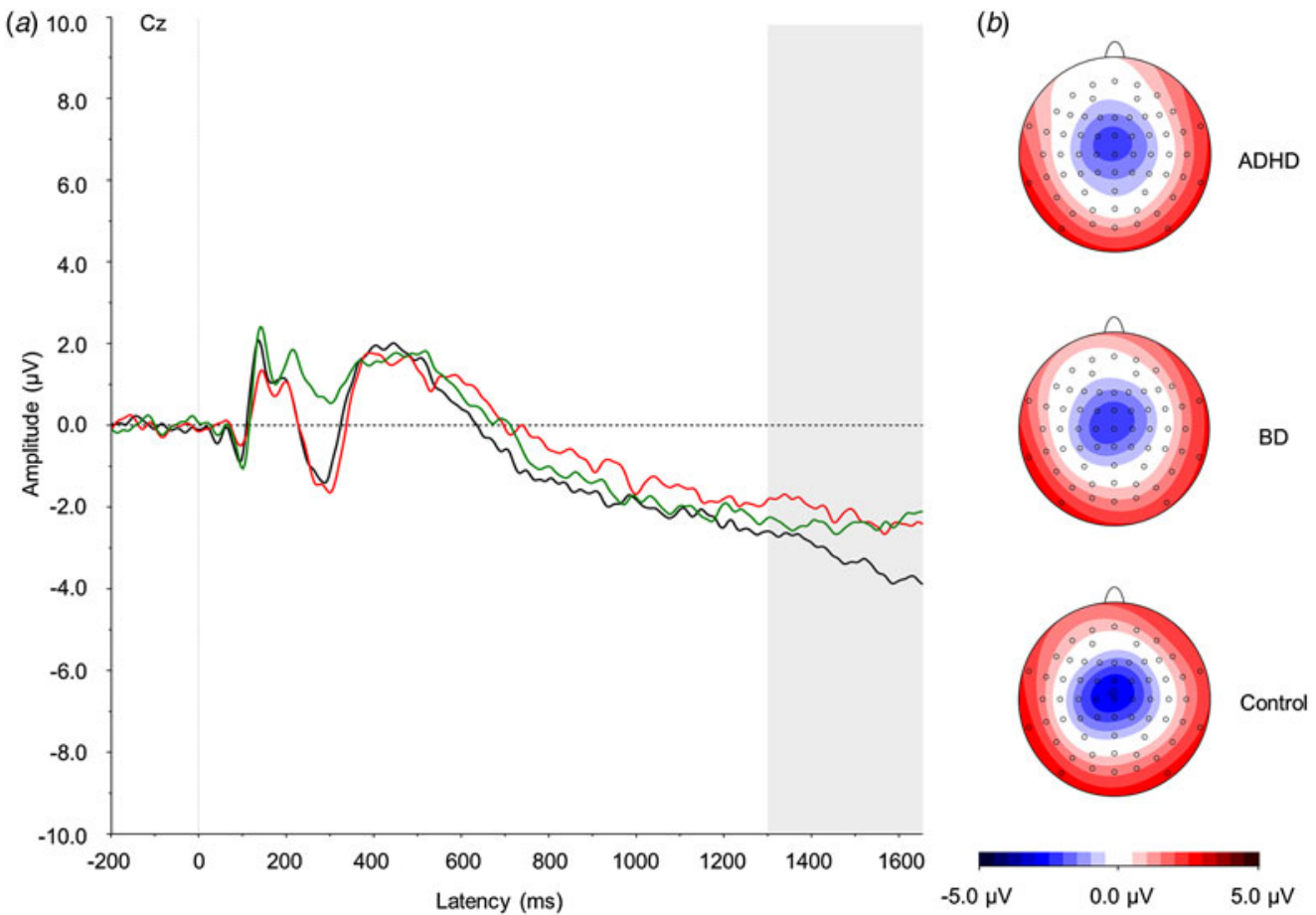

Fig. 1. (a) Grand average event-related potentials to cue stimuli at the $\mathrm{Cz}$ electrode, showing the contingent negative variation in the 1300-1650 ms window. ADHD, Attention-deficit/hyperactivity disorder (light grey; red online); BD, bipolar disorder (mid grey; green online). Controls are shown in black. (b) Topographic maps for each group. For a colour figure, see the online version.
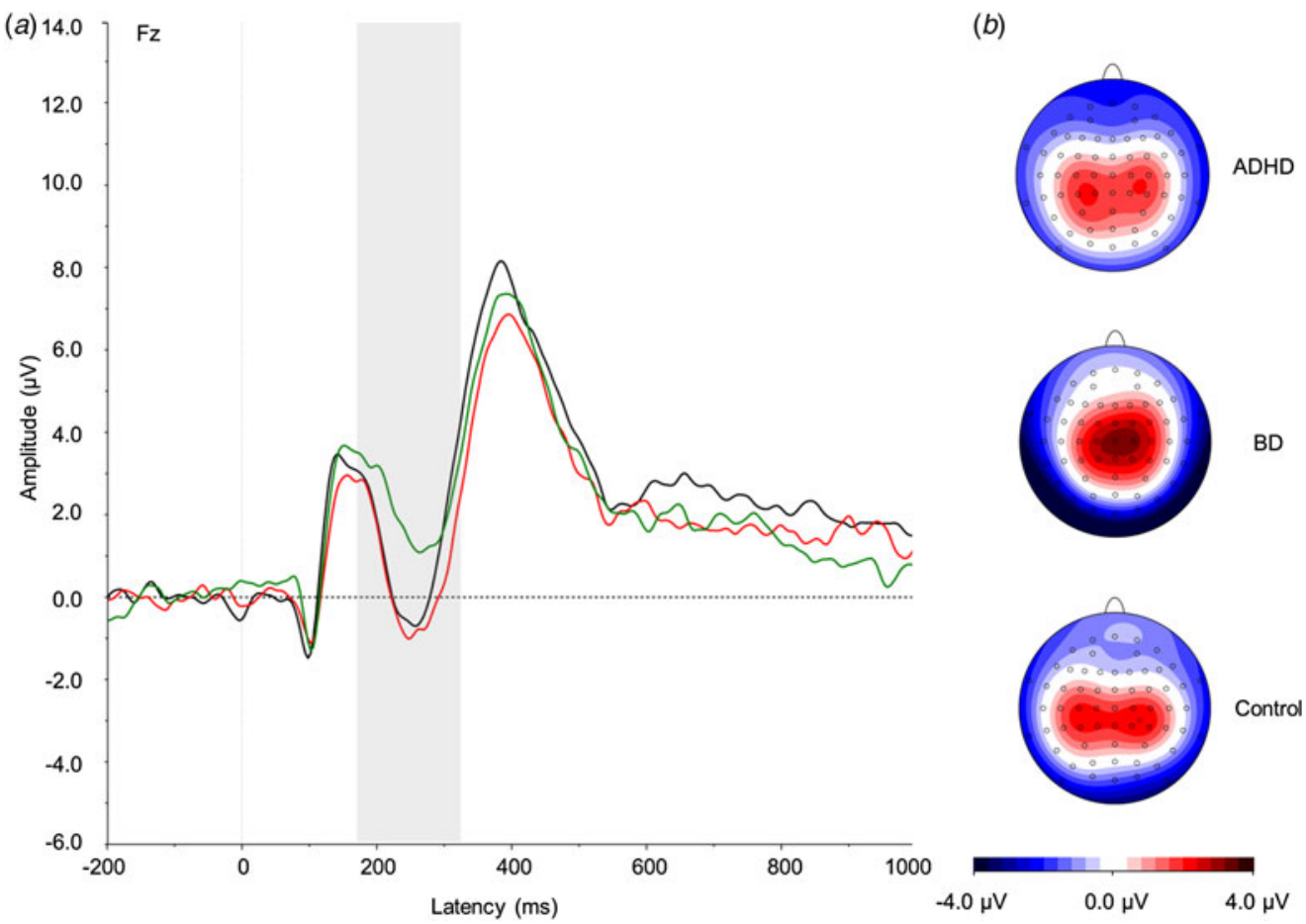

Fig. 2. (a) Grand average event-related potentials to NoGo stimuli at the Fz electrode, showing the NoGo-N2 in the 175-325 ms window. ADHD, Attention-deficit/hyperactivity disorder (light grey; red online); BD, bipolar disorder (mid grey; green online). Controls are shown in black. (b) Topographic maps for each group. For a colour figure, see the online version. 


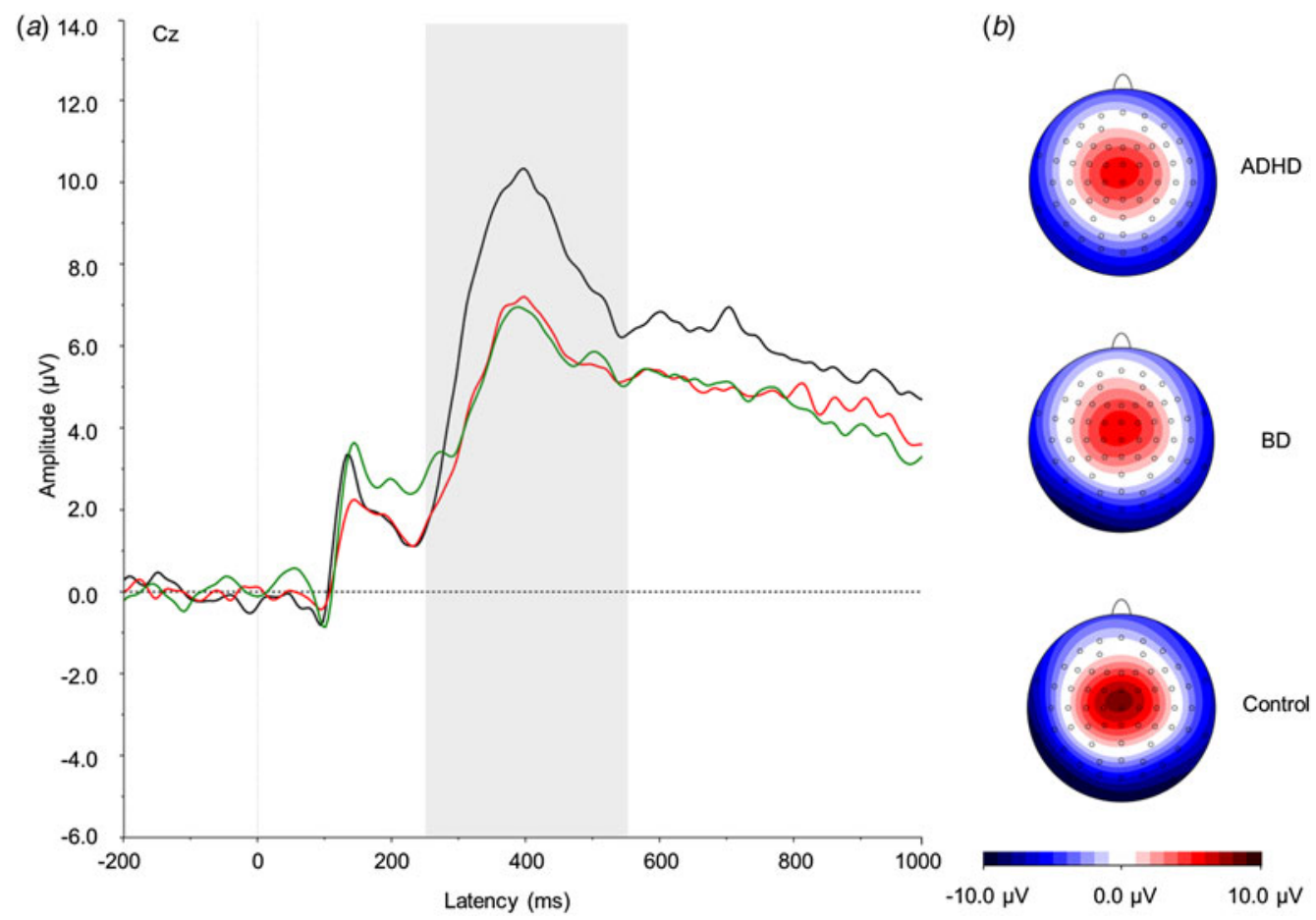

Fig. 3. (a) Grand average event-related potentials to NoGo stimuli at the Cz electrode, showing the NoGo-P3 in the 250-550 ms window. ADHD, Attention-deficit/hyperactivity disorder (light grey; red online); BD, bipolar disorder (mid grey; green online). Controls are shown in black. (b) Topographic maps for each group. For a colour figure, see the online version.

where $d=0.20$ constitutes a small effect, $d=0.50$ a medium effect and $d=0.80$ a large effect (Cohen, 1988).

\section{Ethical standards}

All procedures contributing to this work comply with the ethical standards of the relevant national and institutional committees on human experimentation and with the Helsinki Declaration of 1975, as revised in 2008 .

\section{Results}

\section{Cognitive performance measures}

A trend-level effect of group emerged for RTV $\left(F_{2,57}=\right.$ 2.67, $p=0.08)$. Post-hoc analyses revealed a significant difference between the BD and control groups ( $p=$ 0.03 ) and a trend-level difference between the ADHD and control groups $(p=0.06)$ on RTV, both with medium effect sizes (Table 1), but no differences between the ADHD and BD groups $(p=0.93)$. Groups did not differ on MRT $\left(F_{2,57}=1.47, p=0.24\right)$.

Trend-level effects emerged on the number of total commission errors $\left(H_{2}=4.96, p=0.08\right)$ and omission errors $\left(H_{2}=4.74, p=0.09\right)$. Post-hoc analyses indicated that participants with ADHD made significantly more commission $(p=0.03)$ and omission $(p=0.04)$ errors than controls, with medium and small effect sizes, respectively (Table 1). Participants with BD showed a trend-level difference on the number of omission errors $(p=0.07)$ from controls, with a small effect size, but no difference on commission errors $(p=0.34)$. The ADHD and BD groups did not differ on commission $(p=0.20)$ or omission $(p=0.90)$ errors. No effect of group emerged for OXO-not-XOX commission errors $\left(H_{2}=3.81, p=0.15\right)$.

\section{ERP parameters}

Cue condition

An effect of group did not emerge on the Cue-P3 $\left(F_{2,57}=1.31, p=0.28\right)$.

A trend-level effect of group emerged for the CNV $\left(F_{2,57}=2.86, p=0.07\right)$. Post-hoc comparisons showed a significant difference between the ADHD and control groups $(p=0.05)$ and a trend-level difference between the BD and control groups $(p=0.09)$, both with medium effect size (Table 1$)$. No difference emerged between the two clinical groups $(p=0.85)$.

\section{NoGo condition}

There was a significant effect of group on the NoGo-N2 $\left(F_{2,57}=4.03, p=0.02\right)$. Post-hoc analyses revealed that the $\mathrm{BD}$ group significantly differed from the ADHD 
Table 1. Cognitive performance and event-related potential measures from the cued continuous performance test: means, effect sizes and significance of group comparisons

\begin{tabular}{|c|c|c|c|c|c|c|}
\hline & $\begin{array}{l}\operatorname{ADHD}(n=20)^{\mathrm{a}}: \\
\text { mean (s.D.) }\end{array}$ & $\begin{array}{l}\mathrm{BD}(n=20): \\
\text { mean }(\text { s.D. })^{\mathrm{b}}\end{array}$ & $\begin{array}{l}\text { Controls }(n=20) \text { : } \\
\text { mean (S.D. })^{\mathrm{b}}\end{array}$ & $\begin{array}{l}\text { ADHD } v . \\
\text { BD, effect } \\
\text { size: } d\end{array}$ & $\begin{array}{l}\text { ADHD } v \text {. } \\
\text { controls, effect } \\
\text { size: } d\end{array}$ & $\begin{array}{l}\text { BD } v \text {. controls, } \\
\text { effect size: } d\end{array}$ \\
\hline MRT, ms & $425.31(75.74)$ & $418.30(67.41)$ & $391.58(63.68)$ & 0.05 & $0.49^{\mathrm{d}}$ & 0.44 \\
\hline $\mathrm{RTV}, \mathrm{ms}$ & $109.18(58.83)$ & $101.73(37.77)$ & $76.91(39.24)$ & 0.02 & $0.60^{\mathrm{d}}+$ & $0.68^{\mathrm{d} *}$ \\
\hline OE & $1.10(1.55)$ & $1.35(2.52)$ & $0.60(1.57)$ & 0.12 & $0.32^{*}$ & $0.36+$ \\
\hline $\begin{array}{l}\text { OXO-not-XOX } \\
\text { CE }\end{array}$ & $1.05(1.88)$ & $0.60(2.04)$ & $0.50(0.89)$ & 0.23 & 0.37 & 0.06 \\
\hline Total CE & 7.25 (16.03) & $2.40(5.39)$ & $0.75(0.97)$ & 0.41 & $0.57^{\mathrm{d}_{*}}$ & 0.43 \\
\hline Cue-P3 at Pz & $2.30(1.64)$ & $1.36(1.80)$ & $1.83(2.04)$ & $0.56^{\mathrm{d}}$ & 0.26 & 0.25 \\
\hline $\mathrm{CNV}$ at $\mathrm{Cz}$ & $-2.24(1.03)$ & $-2.31(1.36)$ & $-3.31(2.12)$ & 0.06 & $0.66^{\mathrm{d} *}$ & $0.58^{\mathrm{d}_{\dagger}}$ \\
\hline NoGo-N2 at Fz & 0.57 (1.88) & $2.41(2.64)$ & $0.84(2.07)$ & $0.83^{\mathrm{c} *}$ & 0.14 & $0.68^{\mathrm{d} *}$ \\
\hline NoGo-P3 at Cz & $5.42(2.73)$ & $5.56(3.31)$ & $7.68(2.57)$ & 0.05 & $0.88^{\mathrm{c} *}$ & $0.73^{\mathrm{d} *}$ \\
\hline Go-P3 at $\mathrm{CPz}$ & $5.01(2.76)$ & $5.56(3.23)$ & $6.10(2.18)$ & 0.19 & $0.45^{\mathrm{d}}$ & 0.20 \\
\hline
\end{tabular}

ADHD, Attention-deficit/hyperactivity disorder; S.D., standard deviation; BD, bipolar disorder; MRT, mean reaction time; $\mathrm{RTV}$, within-subject variability in reaction times; $\mathrm{OE}$, omission errors; $\mathrm{CE}$, commission errors; $\mathrm{CNV}$, contingent negative variation

${ }^{\text {a }}$ Only 18 participants with ADHD were included in the average of the Go condition, as two participants did not have at least 20 artefact-free segments.

${ }^{\mathrm{b}}$ Means and S.D.s were calculated on raw data.

${ }^{\mathrm{c}}$ Large effect size.

${ }^{\mathrm{d}}$ Medium effect size.

$* p<0.05,+p<0.10$.

$(p=0.015)$ and control $(p=0.04)$ groups, with large and medium effect sizes, respectively (Table 1). The ADHD and control groups did not differ from each other $(p=0.66)$.

A significant effect of group emerged on the NoGo-P3 $\quad\left(F_{2,57}=3.86, \quad p=0.03\right)$. Post-hoc analyses showed that both the ADHD $(p=0.01)$ and BD $(p=$ 0.03 ) groups significantly differed from controls, respectively, with large and medium effect sizes (Table 1), but not from each other $(p=0.88)$.

\section{Go condition}

No significant effect of group emerged on the Go-P3 $\left(F_{2,55}=0.73, p=0.49\right)$.

\section{Discussion}

In a direct comparison of women with ADHD, women with $\mathrm{BD}$ and control women on cognitive performance and ERP measures from a CPT-OX task, we report evidence for both disorder-specific (conflict monitoring) and overlapping (inhibitory control and potentially response preparation) neurophysiological impairments across the disorders. The current study represents the first cognitive-electrophysiological investigation comparing attentional and inhibitory processing in adults with ADHD and adults with BD. In addition, since the majority of previous ERP studies on ADHD have used male samples (McLoughlin et al. 2010, 2011; Albrecht et al. 2013; Doehnert et al. 2013), and very few studies of this kind have been conducted in BD, our all-female sample furthers our understanding of neurophysiological impairments in females with either of these disorders.

Our ERP results show a significant difference between the ADHD and BD groups in the amplitude of the N2 in response to NoGo stimuli, which was reduced in participants with $\mathrm{BD}$ compared with the other two groups. The N2 is considered to reflect conflict-monitoring processing (Holroyd et al. 2003; Yeung \& Cohen, 2006) and to depend on the amount of correct response processing needed to overcome a conflicting response. In the CPT-OX, this process may be represented by the bias towards the response after a cue, which requires the preparation of a response, and produces increased conflict monitoring when the prepared response has to be stopped in presence of a non-target. The reduced N2 in women with BD aligns with previous evidence of attenuated N2 elicited with an oddball task (Ethridge $e$ t al. 2012) and of a reduced ERN in error responses (Morsel et al. 2014). Both N2 and ERN in conditions inducing conflict, such as in non-target or incongruent trials, are thought to reflect 
conflict monitoring (Yeung \& Cohen, 2006). Our results may therefore indicate that women with BD show impaired conflict monitoring compared with women with ADHD and control women. In line with previous studies using the CPT-OX (McLoughlin et al. 2010, 2011; Albrecht et al. 2013; Doehnert et al. 2013), we did not find an attenuated NoGo-N2 in women with ADHD, although reduced N2 have been associated with ADHD in tasks inducing higher conflict demands (McLoughlin et al. 2009, 2014b).

We also identified abnormalities in ERPs that distinguished women in both clinical groups from controls, indicating shared neurophysiological impairments across ADHD and BD. The reduced P3 in response to NoGo stimuli in both ADHD and BD groups, compared with the control group, suggests a similar pattern of impaired response inhibition to that previously reported in investigations of children and adults with ADHD (McLoughlin et al. 2010, 2011; Albrecht et al. 2013; Doehnert et al. 2013). The reduced NoGo-P3 in women with BD also aligns with previous cognitive research finding deficits in inhibitory control in euthymic BD (Robinson et al. 2006, 2013). These attenuations of the NoGo-P3 in both disorders therefore probably represent an area of overlapping impairment in brain processes implicated in the inhibition of incorrect response. Yet, this inhibitory control deficit in women with BD was temporally preceded by other processing deficits in the NoGo-N2. As such, in ERPs to non-targets, while women with ADHD seem primarily impaired in response inhibition, women with BD show a broader deficit in both conflict monitoring and inhibitory control.

Additionally, we report an attenuation in the CNV in women with ADHD compared with controls, and also potentially in women with BD (trend-level difference), both with a medium effect size. These results replicate previous studies reporting reduced $\mathrm{CNV}$ in individuals with ADHD (McLoughlin et al. 2010, 2011; Albrecht et al. 2013; Doehnert et al. 2013; Tye et al. 2014), and suggest another potential area of shared impairment with BD. However, we note that the comparison between $\mathrm{BD}$ and control participants was only at trend level. If replicated also in $\mathrm{BD}$, this attenuation of the CNV would index an overlapping impairment in response preparation in the two disorders.

The lack of a difference between women with ADHD and controls in the Cue-P3 is inconsistent with some previous investigations showing a reduced Cue-P3 in ADHD samples (McLoughlin et al. 2010; Albrecht et al. 2013). Yet, these attenuations have not been reported in all studies (Dhar et al. 2010; Skirrow, 2012) and the difference in the Cue-P3 emerged as significant, but with a small effect size, in a recent larger-scale study of adolescents and young adults with ADHD (Cheung et al. 2015). In the present study, the normal Cue-P3 in ADHD may be due to an effect of gender, the current study being the first using an all-female sample. An age-effect is also plausible, since this study included adults of a slightly older and broader age range compared with previous investigations (e.g. McLoughlin et al. 2010) and developmental changes have been reported for the Cue-P3, suggesting that ADHD-control differences may decline with age (Doehnert et al. 2013). Further studies on larger samples that include participants of both genders and a broader age range are needed to clarify potential gender and age effects on these processes in ADHD.

While ERP measures of conflict monitoring differentiated the ADHD and BD groups, cognitive performance data did not suggest differences between the two clinical groups. Our cognitive performance results potentially suggest poorer performance and higher RTV in both ADHD and BD groups, compared with controls, consistent with previous studies reporting lower accuracy and higher RTV in ADHD and BD independently (Brotman et al. 2009; Kuntsi et al. 2010; Torralva et al. 2011). This pattern of results, with differences between ADHD and BD groups observed in the neurophysiological markers but not at the cognitive performance level, may reflect greater specificity of the neurophysiological markers in detecting differences between clinical groups.

The following limitations of this study should be taken into account when interpreting these data. First, although the groups were matched on gender, age and IQ, there were differences in the prescribed medications that participants with ADHD or BD were taking. While we asked participants with ADHD to stop taking stimulant medications $48 \mathrm{~h}$ prior to the assessment, it was not possible, for ethical reasons, to ask participants to stop mood-stabilizing, anti-psychotic or antidepressant medications. Given limited numbers in medication subgroups, we were not able to directly test the effect of medication on ERP measures, which represents a limitation of the current study. The effects of medication are difficult to control for in cross-disorder comparison studies where different treatments may be prescribed to different groups of psychiatric patients. Although the understanding of the effects of medications on ERPs is still limited, previous studies suggest that medications may normalize ERP measures (Anderer et al. 2002; Karaaslan et al. 2003; Galletly et al. 2005). As such, in this study, a medication effect could potentially have resulted in ERPs comparable with controls. Yet, both clinical groups, although some participants were medicated, showed reduced ERP measures compared with controls. Therefore, although the effect of medication 
represents a potential confounder of this study and may have attenuated some case-control differences, we report impairments in both clinical groups which may not have been produced by the effect of medication. Future studies on samples including non-medicated individuals or a higher number of individuals in each medication subgroup are needed to clarify whether our results may have been affected by medication effects. A second limitation is that, by using an area measure, we were not able to obtain latency data. This approach, previously adopted in similar ERP studies (Groom et al. 2010; Tye et al. 2014), was preferred for having the advantage, over peak measures, of being unaffected by latency variability and of providing a reliable measure of amplitude even when the identification of clear peaks is not possible for all subjects (Luck, 2005). Although some previous studies found prolonged latency of ERP components in BD (Chun et al. 2013; Maekawa et al. 2013), our ERP grand averages did not suggest latency differences, thus our area measure probably captured most of the differences between the groups on ERP measures. Finally, in order to increase homogeneity of the sample, this investigation was conducted on an all-female sample, with slightly higher than expected IQ in the clinical groups. Replication in future investigations with bigger samples of both genders and including individuals with a wider range of IQs is required in order to generalize these findings to more typical clinical populations.

\section{Conclusion}

In conclusion, our results represent some of the first evidence of disorder-specific and shared impairments in brain processes involved in attentional orienting, conflict monitoring and inhibitory control in women with ADHD and BD, with moderate to large effect sizes. This investigation of neurophysiological processes furthers our understanding of impairments associated with ADHD and BD, and the identification of objective measures showing differences between $\mathrm{ADHD}$ and BD may assist in differentiating between the two disorders when their distinction is not clear at clinical consultations. If replicated in larger-scale studies, the neurophysiological biomarkers of distinct patterns in brain activity may aid in the identification of the diagnostic boundaries of ADHD and BD in adults. More broadly, given that ADHD and BD are both highly heritable disorders, the identified neurophysiological indices may represent intermediate phenotypes between diagnosis and genetic factors influencing a disorder, as suggested by genetic and family studies on ERP indices of attentional and inhibitory processing showing shared familial/genetic influences with ADHD (McLoughlin et al. 2011;
Albrecht et al. 2013, 2014). Future studies can investigate causal models of ADHD and BD, by exploring to what extent overlapping and disorder-specific impairments in brain function are accounted for by specific or shared genetic influences on the two disorders and, in turn, further our understanding on the pathways to distinct and overlapping features in ADHD and BD.

\section{Supplementary material}

For supplementary material accompanying this paper visit http://dx.doi.org/10.1017/S0033291715001877

\section{Acknowledgements}

G. Michelini is supported by a $1+3$ Ph.D. studentship awarded by the Medical Research Council (MRC) Social, Genetic and Developmental Psychiatry Centre, Institute of Psychiatry, Psychology and Neuroscience, King's College London (G9817803). This project was supported by an Economic and Social Research Council studentship to G. L. Kitsune (ES/100971X/1). This paper represents independent research part funded by the National Institute for Health Research (NIHR) Biomedical Research Centre and Dementia Unit at South London and Maudsley National Health Service (NHS) Foundation Trust and King's College London. The views expressed are those of the authors and not necessarily those of the NHS, the NIHR or the Department of Health. We thank all who make this research possible: The National Adult ADHD Clinic at the South London and Maudsley Hospital, Dr Helen Costello, Professor Sophia Frangou, Professor Anne Farmer, Jessica Deadman, Hannah Collyer, Sarah-Jane Gregori and all participants who contributed their time to the study.

\section{Declaration of Interest}

P. Asherson has received funding for research by Vifor Pharma, and has given sponsored talks and been an advisor for Shire, Janssen-Cilag, Eli-Lilly, Flynn Pharma and Pfizer, regarding the diagnosis and treatment of ADHD. All funds are received by King's College London and used for studies of ADHD. The other authors report no conflicts of interest.

\section{Note}

${ }^{1}$ Since most previous ERP analyses on CPT-OX did not apply a baseline subtraction (Banaschewski et al. 2004; McLoughlin et al. 2010, 2011; Albrecht et al. 2013; Doehnert et al. 2013), analyses were also repeated without baseline correction. Results of data without baseline 
correction were comparable for the NoGo-N2, NoGo-P3 and Go-P3, but partly changed for the Cue-P3 and CNV (see Supplementary material).

\section{References}

Albrecht B, Brandeis D, Uebel H, Valko L, Heinrich H, Drechsler R, Heise A, Müller UC, Steinhausen HC, Rothenberger A, Banaschewski T (2013). Familiality of neural preparation and response control in childhood attention deficit-hyperactivity disorder. Psychological Medicine 43, 1997-2011.

Albrecht B, Brandeis D, Uebel-von Sandersleben H, Valko L, Heinrich H, Xu X, Drechsler R, Heise A, Kuntsi J, Müller UC, Asherson P, Steinhausen HC, Rothenberger A, Banaschewski T (2014). Genetics of preparation and response control in ADHD: the role of DRD4 and DAT1. Journal of Child Psychology and Psychiatry 55, 914-923.

Altman EG, Hedeker D, Peterson JL, Davis JM (1997). The Altman Self-Rating Mania Scale. Biological Psychiatry 42, 948-955.

Anderer P, Saletu B, Semlitsch HV, Pascual-Marqui RD (2002). Perceptual and cognitive event-related potentials in neuropsychopharmacology: methodological aspects and clinical applications (pharmaco-ERP topography and tomography). Methods and Findings in Experimental and Clinical Pharmacology 24, 121-137.

APA (2000). Diagnostic and Statistical Manual of Mental Disorders. American Psychiatric Association: Washington, DC.

Asherson P, Young AH, Eich-Höchli D, Moran P, Porsdal V, Deberdt W (2014). Differential diagnosis, comorbidity, and treatment of attention-deficit/hyperactivity disorder in relation to bipolar disorder or borderline personality disorder in adults. Current Medical Research and Opinion 30, 1657-1672.

Banaschewski T, Brandeis D (2007). Annotation: what electrical brain activity tells us about brain function that other techniques cannot tell us - a child psychiatric perspective. Journal of Child Psychology and Psychiatry 48, 415-435.

Banaschewski T, Brandeis D, Heinrich H, Albrecht B, Brunner E, Rothenberger A (2004). Questioning inhibitory control as the specific deficit of ADHD - evidence from brain electrical activity. Journal of Neural Transmission 111, 841-864.

Barry RJ, Clarke AR, McCarthy R, Selikowitz M, Brown CR, Heaven PC (2009). Event-related potentials in adults with attention-deficit/hyperactivity disorder: an investigation using an inter-modal auditory/visual oddball task. International Journal of Psychophysiology 71, 124-131.

Beck AT, Steer RA, Ball R, Ranieri WF (1996). Comparison of Beck Depression Inventories-IA and -II in psychiatric outpatients. Journal of Personality Assessment 67, 588-597.

Bestelmeyer PE (2012). The visual P3a in schizophrenia and bipolar disorder: effects of target and distractor stimuli on the P300. Psychiatry Research 197, 140-144.

Bokura H, Yamaguchi S, Kobayashi S (2001). Electrophysiological correlates for response inhibition in a Go/NoGo task. Clinical Neurophysiology 112, 2224-2232.
Brotman MA, Rooney MH, Skup M, Pine DS, Leibenluft E (2009). Increased intrasubject variability in response time in youths with bipolar disorder and at-risk family members. Journal of the American Academy of Child and Adolescent Psychiatry 48, 628-635.

Cabranes JA, Ancín I, Santos JL, Sánchez-Morla E, García-Jiménez MA, Rodríguez-Moya L, Fernández C, Barabash A (2013). P50 sensory gating is a trait marker of the bipolar spectrum. European Neuropsychopharmacology 23, 721-727.

Cheung CHM, Rijsdijk F, McLoughlin G, Brandeis D, Banaschewski T, Asherson P, Kuntsi J (2015). Cognitive and neurophysiological markers of ADHD persistence and remission. British Journal of Psychiatry. Published online 6 August 2015. doi:10.1192/bjp.bp.114.145185.

Chun J, Karam ZN, Marzinzik F, Kamali M, O'Donnell L, Tso IF, Manschreck TC, McInnis M, Deldin PJ (2013). Can P300 distinguish among schizophrenia, schizoaffective and bipolar I disorders? An ERP study of response inhibition. Schizophrenia Research 151, 175-184.

Cohen J (1988). Statistical Power Analysis for the Behavioral Sciences, 2nd edn. Lawrence Erlbaum Associates: Hillsdale, NJ.

Das D, Cherbuin N, Butterworth P, Anstey KJ, Easteal S (2012). A population-based study of attention deficit/ hyperactivity disorder symptoms and associated impairment in middle-aged adults. PLOS One 7, e31500.

Dhar M, Been PH, Minderaa RB, Althaus M (2010). Information processing differences and similarities in adults with dyslexia and adults with attention deficit hyperactivity disorder during a continuous performance test: a study of cortical potentials. Neuropsychologia 48, 3045-3056.

Doehnert M, Brandeis D, Imhof K, Drechsler R, Steinhausen HC (2010). Mapping attention-deficit/ hyperactivity disorder from childhood to adolescence - no neurophysiologic evidence for a developmental lag of attention but some for inhibition. Biological Psychiatry 67, 608-616.

Doehnert M, Brandeis D, Schneider G, Drechsler R, Steinhausen HC (2013). A neurophysiological marker of impaired preparation in an 11-year follow-up study of attention-deficit/hyperactivity disorder (ADHD). Journal of Child Psychology and Psychiatry 54, 260-270.

Doehnert M, Brandeis D, Straub M, Steinhausen HC, Drechsler R (2008). Slow cortical potential neurofeedback in attention deficit hyperactivity disorder: is there neurophysiological evidence for specific effects? Journal of Neural Transmission 115, 1445-1456.

Ethridge LE, Hamm JP, Shapiro JR, Summerfelt AT, Keedy SK, Stevens MC, Pearlson G, Tamminga CA, Boutros NN, Sweeney JA, Keshavan MS, Thaker G, Clementz BA (2012). Neural activations during auditory oddball processing discriminating schizophrenia and psychotic bipolar disorder. Biological Psychiatry 72, 766-774.

Faraone SV, Biederman J (2005). What is the prevalence of adult ADHD? Results of a population screen of 966 adults. Journal of Attention Disorders 9, 384-391.

Gajewski PD, Falkenstein M (2013). Effects of task complexity on ERP components in Go/Nogo tasks. International Journal of Psychophysiology 87, 273-278. 
Galletly CA, Clark CR, McFarlane AC (2005). Clozapine improves working memory updating in schizophrenia. European Neuropsychopharmacology 15, 601-608.

Groom MJ, Scerif G, Liddle PF, Batty MJ, Liddle EB, Roberts KL, Cahill JD, Liotti M, Hollis C (2010). Effects of motivation and medication on electrophysiological markers of response inhibition in children with attention-deficit/ hyperactivity disorder. Biological Psychiatry 67, 624-631.

Hall MH, Rijsdijk F, Kalidindi S, Schulze K, Kravariti E, Kane F, Sham P, Bramon E, Murray RM (2007). Genetic overlap between bipolar illness and event-related potentials. Psychological Medicine 37, 667-678.

Henry BL, Minassian A, Perry W (2013). Everyday functional ability across different phases of bipolar disorder. Psychiatry Research 210, 850-856.

Holroyd CB, Nieuwenhuis S, Yeung N, Cohen JD (2003). Errors in reward prediction are reflected in the event-related brain potential. Neuroreport 14, 2481-2484.

Hosang GM, Uher R, Maughan B, McGuffin P, Farmer AE (2012). The role of loss and danger events in symptom exacerbation in bipolar disorder. Journal of Psychiatric Research 46, 1584-1589.

Ibanez A, Cetkovich M, Petroni A, Urquina H, Baez S, Gonzalez-Gadea ML, Kamienkowski JE, Torralva T, Torrente F, Strejilevich S, Teitelbaum J, Hurtado E, Guex R, Melloni M, Lischinsky A, Sigman M, Manes F (2012). The neural basis of decision-making and reward processing in adults with euthymic bipolar disorder or attention-deficit/hyperactivity disorder (ADHD). PLOS ONE 7, e37306.

Jahshan C, Wynn JK, Mathis KI, Altshuler LL, Glahn DC, Green MF (2012). Cross-diagnostic comparison of duration mismatch negativity and P3a in bipolar disorder and schizophrenia. Bipolar Disorders 14, 239-248.

Jung TP, Makeig S, Humphries C, Lee TW, McKeown MJ, Iragui V, Sejnowski TJ (2000). Removing electroencephalographic artifacts by blind source separation. Psychophysiology 37, 163-178.

Karaaslan F, Gonul AS, Oguz A, Erdinc E, Esel E (2003). P300 changes in major depressive disorders with and without psychotic features. Journal of Affective Disorders 73, 283-287.

Kooij JJS, Francken MH (2007). Diagnostic Interview for ADHD in Adults (DIVA) (http://www.psyq.nl/files/ 1263005/DIVA_2_EN.pdf). Accessed September 2015.

Kuntsi J, Klein C (2012). Intraindividual variability in ADHD and its implications for research of causal links. Current Topics in Behavioral Neurosciences 9, 67-91.

Kuntsi J, Wood AC, Rijsdijk F, Johnson KA, Andreou P, Albrecht B, Arias-Vasquez A, Buitelaar JK, McLoughlin G, Rommelse NN, Sergeant JA, Sonuga-Barke EJ, Uebel H, van der Meere JJ, Banaschewski T, Gill M, Manor I, Miranda A, Mulas F, Oades RD, Roeyers H, Rothenberger A, Steinhausen HC, Faraone SV, Asherson P (2010). Separation of cognitive impairments in attention-deficit/ hyperactivity disorder into 2 familial factors. Archives of General Psychiatry 67, 1159-1167.

Luck SJ (2005). An Introduction to the Event-Related Potential Technique. MIT Press: Cambridge, MA.
Maekawa T, Katsuki S, Kishimoto J, Onitsuka T, Ogata K, Yamasaki T, Ueno T, Tobimatsu S, Kanba S (2013). Altered visual information processing systems in bipolar disorder: evidence from visual MMN and P3. Frontiers in Human Neuroscience 7, 403.

McLoughlin G, Albrecht B, Banaschewski T, Rothenberger A, Brandeis D, Asherson P, Kuntsi J (2009). Performance monitoring is altered in adult ADHD: a familial event-related potential investigation. Neuropsychologia 47, 3134-3142.

McLoughlin G, Albrecht B, Banaschewski T, Rothenberger A, Brandeis D, Asherson P, Kuntsi J (2010). Electrophysiological evidence for abnormal preparatory states and inhibitory processing in adult ADHD. Behavioral and Brain Functions 6, 66.

McLoughlin G, Asherson P, Albrecht B, Banaschewski T, Rothenberger A, Brandeis D, Kuntsi J (2011). Cognitiveelectrophysiological indices of attentional and inhibitory processing in adults with ADHD: familial effects. Behavioral and Brain Functions 7, 26.

McLoughlin G, Makeig S, Tsuang MT (2014a). In search of biomarkers in psychiatry: EEG-based measures of brain function. American Journal of Medical Genetics Part B: Neuropsychiatric Genetics 165B, 111-121.

McLoughlin G, Palmer JA, Rijsdijk F, Makeig S (2014b). Genetic overlap between evoked frontocentral theta-band phase variability, reaction time variability, and attention-deficit/hyperactivity disorder symptoms in a twin study. Biological Psychiatry 75, 238-247.

Merikangas KR, He JP, Burstein M, Swendsen J, Avenevoli S, Case B, Georgiades K, Heaton L, Swanson S, Olfson M (2011). Service utilization for lifetime mental disorders in US adolescents: results of the National Comorbidity SurveyAdolescent Supplement (NCS-A). Journal of the American Academy of Child and Adolescent Psychiatry 50, 32-45.

Morsel AM, Morrens M, Temmerman A, Sabbe B, de Bruijn ER (2014). Electrophysiological (EEG) evidence for reduced performance monitoring in euthymic bipolar disorder. Bipolar Disorders 16, 820-829.

Pini S, de Queiroz V, Pagnin D, Pezawas L, Angst J, Cassano GB, Wittchen HU (2005). Prevalence and burden of bipolar disorders in European countries. European Neuropsychopharmacology 15, 425-434.

Robinson LJ, Thompson JM, Gallagher P, Goswami U, Young AH, Ferrier IN, Moore PB (2006). A meta-analysis of cognitive deficits in euthymic patients with bipolar disorder. Journal of Affective Disorders 93, 105-115.

Robinson LJ, Thompson JM, Gallagher P, Gray JM, Young AH, Ferrier IN (2013). Performance monitoring and executive control of attention in euthymic bipolar disorder: employing the CPT-AX paradigm. Psychiatry Research 210, 457-464.

Schulze KK, Hall MH, McDonald C, Marshall N, Walshe M, Murray RM, Bramon E (2008). Auditory P300 in patients with bipolar disorder and their unaffected relatives. Bipolar Disorders 10, 377-386.

Skirrow C (2012). Investigating Emotional Lability in Adults with Attention Deficit Hyperactivity Disorder: An Integrative Approach. Ph.D. Thesis, King's College London. 
Skirrow C, Ebner-Priemer U, Reinhard I, Malliaris Y, Kuntsi J, Asherson P (2014). Everyday emotional experience of adults with attention deficit hyperactivity disorder: evidence for reactive and endogenous emotional lability. Psychological Medicine 44, 3571-3583.

Skirrow C, Hosang GM, Farmer AE, Asherson P (2012). An update on the debated association between ADHD and bipolar disorder across the lifespan. Journal of Affective Disorders 141, 143-159.

Swann AC, Lijffijt M, Lane SD, Steinberg JL, Acas MD, Cox B, Moeller FG (2013). Pre-attentive information processing and impulsivity in bipolar disorder. Journal of Psychiatric Research 47, 1917-1924.

Torralva T, Gleichgerrcht E, Torrente F, Roca M, Strejilevich SA, Cetkovich M, Lischinsky A, Manes F (2011). Neuropsychological functioning in adult bipolar disorder and ADHD patients: a comparative study. Psychiatry Research 186, 261-266.

Torres IJ, Boudreau VG, Yatham LN (2007).

Neuropsychological functioning in euthymic bipolar disorder: a meta-analysis. Acta Psychiatrica Scandinavica 116, $17-26$.

Tye C, Asherson P, Ashwood KL, Azadi B, Bolton P, McLoughlin G (2014). Attention and inhibition in children with ASD, ADHD and co-morbid ASD + ADHD: an event-related potential study. Psychological Medicine 44, 1101-1116.

Valko L, Doehnert M, Müller UC, Schneider G, Albrecht B, Drechsler R, Maechler M, Steinhausen HC, Brandeis D (2009). Differences in neurophysiological markers of inhibitory and temporal processing deficits in children and adults with ADHD. Journal of Psychophysiology 23, 235-246.

van Leeuwen TH, Steinhausen HC, Overtoom CC, Pascual-Marqui RD, van't Klooster B, Rothenberger A, Sergeant JA, Brandeis D (1998). The continuous performance test revisited with neuroelectric mapping: impaired orienting in children with attention deficits. Behavioral and Brain Functions 94, 97-110.

Wechsler D (1999). Wechsler Abbreviated Scale of Intelligence (WASI). Harcourt Assessment: San Antonio, TX.

Willcutt EG (2012). The prevalence of DSM-IV attention-deficit/hyperactivity disorder: a meta-analytic review. Neurotherapeutics 9, 490-499.

Yeung N, Cohen JD (2006). The impact of cognitive deficits on conflict monitoring. Predictable dissociations between the error-related negativity and N2. Psychological Science 17, 164-171.

Young RC, Biggs JT, Ziegler VE, Meyer DA (1978). Rating-scale for mania - reliability, validity and sensitivity. British Journal of Psychiatry 133, 429-435. 